\title{
Harvesting the Full Absorption in Cladding-Pumped Fibers
}

\author{
Michalis N. Zervas ${ }^{1,2}$ \\ (1) Optoelectronics Research Centre, University of Southampton, UK \\ (2) SPI Lasers, Hedge End, Southampton, UK \\ (mnz@orc.soton.ac.uk)
}

\begin{abstract}
We investigate the wavelength and length dependence of the pump absorption along cladding-pumped single as well as coupled multimode fibers showing strong deparures from Beer's law.

OCIS codes: (060.2320); (060.3510) Lasers, fiber; (140.3615) Lasers, ytterbium; (300.1030) Absorption
\end{abstract}

\section{Introduction}

Power scaling in high power fiber lasers has been exclusively based on a number of different cladding pumping schemes. In addition, high brightness pumps (and pump combining arrangements) and high dopant concentrations are required in order to reduce fibre lengths and minimise the deleterious impact of background losses and nonlinear effects. Among the most commonly used cladding-pumping schemes are direct end-pumping (either free-space [1] or through pump combiners [2]) and coupled multimode fibers (GTWave) [3].

It is known that pump absorption in cladding-pumped lasers and amplifiers departs considerably from the expected pure exponential decay (Beer's law) due to inefficient pump mixing [4]-[5]. Such pump power absorption results in non-optimum inversion and can potentially affect severely the performance of the fiber laser. So far, the wavelength dependence of these effects along the fibre length has been largely ignored.

\section{Absorption Wavelength and Length Dependence}

In this talk we investigate experimentally and theoretically the wavelength dependence of the pump absorption along $\mathrm{Yb}^{3+}$-doped fibers, for cladding-pumped single as well as coupled multimode (GTWave) fibers [6]. We show that significant spectral absorption distortions occur along the length with the $976 \mathrm{~nm}$ absorption peak affected the most. We developed a novel theoretical approach, based coupled mode theory, to explain the observed effects. To reduce the number of pump modes (typically $\sim 10^{5}$ ) and make the problem tractable, we have used mode families characterised by the compound mode number $(\mathrm{n}+2 \mathrm{~m})$ of the $L P_{\mathrm{nm}}$ pump modes to approximate their mutual coupling coefficients and overlap with the absorbing core. The evanescent coupling between the lossless modes of the pump fiber and the corresponding attenuated modes of the signal fiber is taken into account and the total power loss has been calculated for different launching conditions.
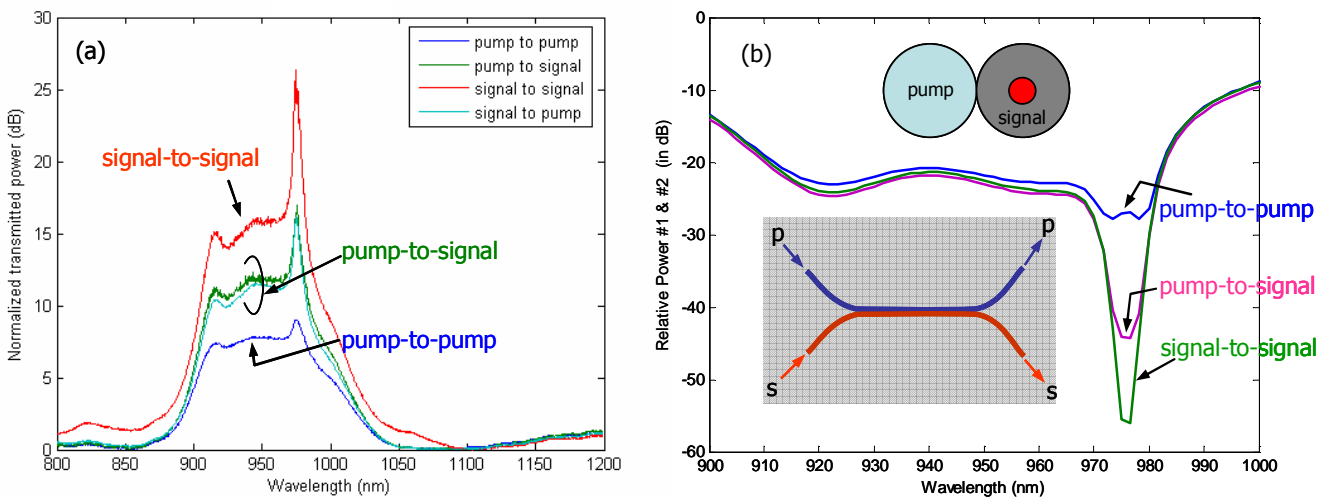

Figure 1: (a) Experimental and (b) theoretical s-s, s-p, p-s \& p-p (s: signal; p: pump) absorption for typical GTWave fiber (the insets in (b) show cross-sectional and longitudinal GTWave details).

Figure 1 shows that the pump absorption in GTWave fibres is highly dependent on the injection and detection ports. It is shown that as a result of the multimode coupling process the pump-to-pump ( $\mathrm{p}-\mathrm{p}$ ) absorption spectrum is substantially reduced and distorted, with the $976 \mathrm{~nm}$ absorption peak suppressed. The s-p and p-s absorption spectra are almost identical, as expected from reciprocity. The s-s absorption spectrum is the strongest and least distorted. These effects are very accurately reproduced by the proposed theory. These differential loss effects are minimised 
by proper mode mixing and/or reduction of the dopant concentration. More details will be given at the conference presentation.

Figure 2(a) shows the variations of the pump-to-pump absorption spectrum as the GTWave length is progressively cut-back. Figure 2(b) plots the length dependence of the absorption at different pump wavelengths. It is shown that the $976 \mathrm{~nm}$ peak absorption wavelength shows the largest departure from Beer's law. Lower absorption wavelengths show progressively smaller departures, with the wavelengths at the absorption wings restoring the Beer-law dependence. Figure 3 plots the corresponding modelling results, showing a very good agreement with experiment.
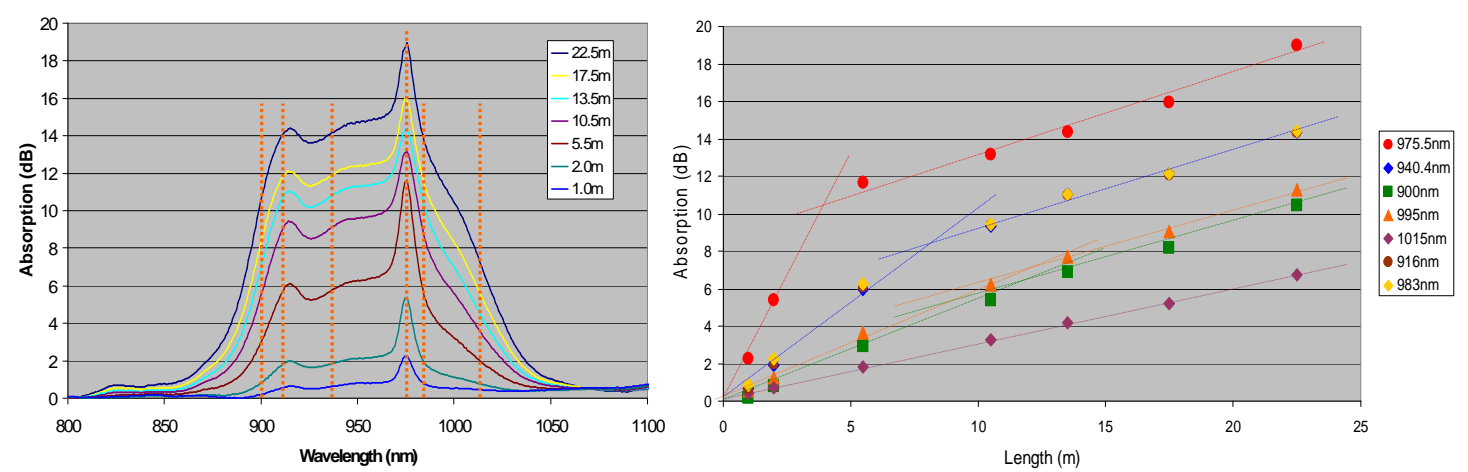

Figure 2: (a) Experimental pump-to pump absorption spectrum as function of GTWave length, (b) p-p absorption as a function of length for different pump wavelengths.
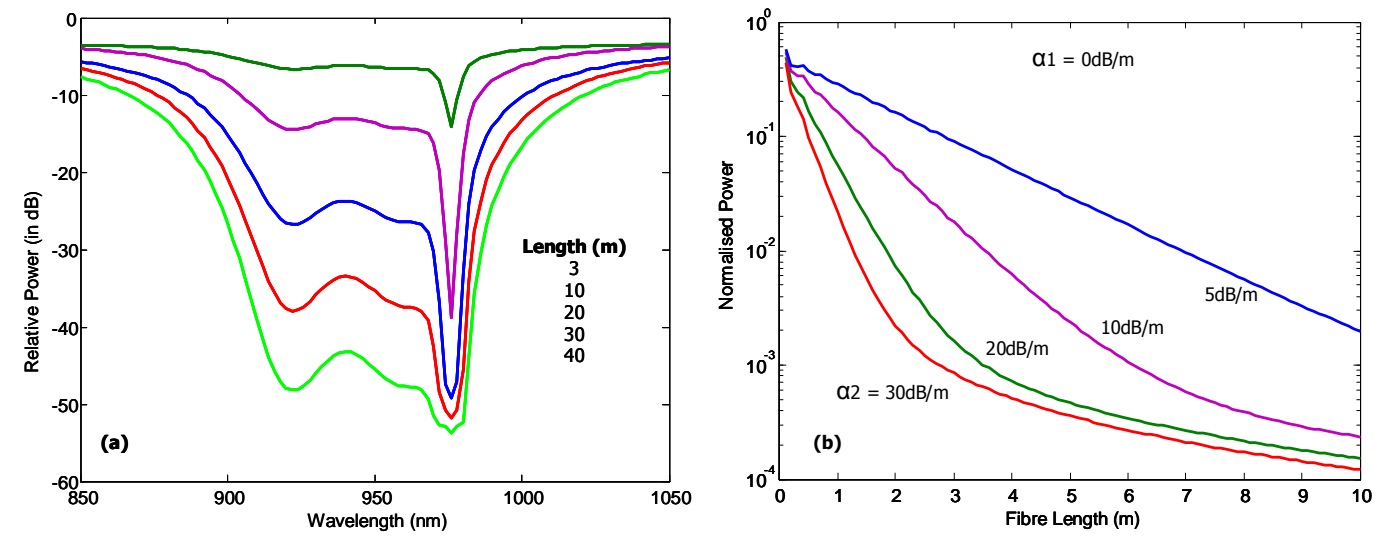

Figure 3: (a) Theoretical pump-to pump absorption spectrum as function of GTWave length, (b) p-p absorption as a function of length for different levels of effective pump loss.

We have also investigated the mode mixing requirements in order to improve the absorption spectral distribution along the length and the increase the overall absorption efficiency. Similar effects and dependencies have been observed in cladding-pumped single Yb-doped fibres. Detailed results will be presented at the conference presentation.

The work was partially supported by the EPSRC Centre for Innovative Manufacturing in Photonics.

\section{References}

[1] Y. Jeong, J. K. Sahu, D. N. Payne, and J. Nilsson, "Ytterbium-doped large-core fiber laser with 1.36 kW continuous-wave output power", Optics Express vol. 12, 6088 (2004).

[2] D. J. DiGiovanni and A. J. Stentz, "Tapered fiber bundles for coupling light into and out of cladding-pumped fiber devices", U.S. patent 5,864,644 (1999)

[3] A.B. Grudinin, D. N. Payne, P. W. Turner, J. Nilsson, M. N. Zervas, M. Ibsen, M.K. Durkin, "Multi-fibre arrangements for high power fibre lasers and amplifiers", US patent 6,826,335 (2000).

[4] S. Bedo, W. Luthy and H.P. Weber, "The effective absorption coefficient in double-clad fibres", Optics Commun. vol. 99, 331 (1993).

[5] V. Doya, O. Legrand and F. Mortessagne, "Optimised absorption in a chaotic double-clad fiber amplifier", Opt. Letters vol. 26, 872 (2001).

[6] M. N. Zervas, A. Marshall, J. Kim, "Effective absorption in cladding-pumped fibers”, Proc. SPIE, vol. 7914, 79141T (2011). 